\section{A QUALITATIVE SCORING SYSTEM OF UNILATERAL LOADING, ASSESSMENT OF MOVEMENT QUALITY IN YOUTH MULTISPORT ATHLETES DURING GROWTH}

\author{
1,2Gemma Parry. ${ }^{1}$ The University of Salford, Manchester, UK; ${ }^{2}$ The English Institute of Sport, \\ Loughborough, UK
}

\subsection{6/bjsports-2021-IOC.290}

Background Movement quality has been identified as an important physical quality within the youth athlete. However, evidence around effects of growth/maturation on movement quality as a risk factor for injury is limited. 3D measures are considered international standard, they are not always practical within the youth environment, feasible field-based alternative methods are required.

Objective To establish validity and reliability of Qualitative Assessment of Single Leg Loading (QASLS) tool in adolescent multisport athletes at different stages of growth

Design Test-Retest Cross Sectional Study.

Setting Field based setting within athlete academy.

Participants 35 (20 male, 15 female) multisport athletes participated in this study.

Interventions 2 unilateral loading tasks were analysed - single leg squat and single leg land - participants were assessed every month for 8-month period performances were evaluated based on their maturational status as determined via peak height velocity (PHV).

Main Outcome Measurements 2D Kinematic Parameters Trunk Lean (Frontal and Sagittal), Hip Adduction Angle, Frontal Plane Projection Angle (FPPA) and Qualitative QASLS Score of movement quality.

Results Results suggest that QASLS and 2D parameters maybe reliability assessed in youth multisport athletes. Within subject compound scoring variation is high. Pre and circa PHV athletes demonstrated greatest variation in movement strategies with highest scores and numbers of strategies used and post PHV the least.

Conclusions This study highlights greater use of increased movement strategies during growth and thus increased prevalence of potential risk factors in younger and athletes encountering the growth spurt. This may have injury, monitoring and return to play implications.

\section{EFFECT OF MATURATION ON KNEE EXTENSOR AND FLEXOR STRENGTH IN MALE AND FEMALE ADOLESCENT ATHLETES}

${ }^{1}$ Kerry Peek, ${ }^{2}$ Kevin Ford, ${ }^{3}$ Gregory Myer, ${ }^{4}$ Timothy Hewett, ${ }^{1}$ Evangelos Pappas. ${ }^{1}$ The University of Sydney, Discipline of Physiotherapy, Sydney, Australia; ${ }^{2}$ High Point University, Department of Physical Therapy, High Point, NC, USA; ${ }^{3}$ SPORT Center, Division of Sports Medicine, Cincinnati Children's Hospital Medical Center" Cincinnati, OH, USA; ${ }^{4}$ Hewett Consulting, Minneapolis, Minnesota, USA

\subsection{6/bjsports-2021-IOC.291}

Background Knee extensor and flexor strength are associated with injury risk, including rupture of the anterior cruciate ligament. However, the longitudinal changes in lower extremity strength with maturation have been underinvestigated.

Objective To investigate the longitudinal changes of knee extension and flexion strength associated with maturation Design The pubertal status [pre-pubertal, pubertal, and postpubertal] was determined with the modified Pubertal
Maturation Observation Scale questionnaire. After a warm-up of five submaximal repetitions, participants were tested for concentric peak isokinetic strength for knee extension and flexion at $300 \%$ s.

Setting Laboratory.

Patients (or Participants) Males and females participating in high school sports who were measured longitudinally in at least two different pubertal stages $(\mathrm{N}=257,208$ females, 18 excluded due to knee injury).

Interventions (or Assessment of Risk Factors) N/A

Main Outcome Measurements Peak knee extension and flexion isokinetic torque was measured across 10 repetitions and normalized to body weight. Separate linear mixed models were used for the right and left side to test for the effect of pubertal stage, sex and their interaction.

Results Significant interactions were identified indicating a different maturational effect on knee muscle strength for males and females, particularly between pre-pubertal and pubertal stages where males demonstrated higher knee extension increases than females $(+12 \%$ vs. $+5 \%$ on right, $+11 \%$ vs $+7 \%$ on left, $\mathrm{p}<0.001$ ) while for knee flexion males demonstrated increased while females demonstrated decreased flexor strength $(+4 \%$ vs. $-1 \%$ on right, $\mathrm{p}=0.03$ and +3 vs. $-3 \%$ on left, $\mathrm{p}=0.009$ ).

Conclusions The findings of this study support a differential effect of maturation on important knee strength outcomes that may have implications for the higher rates of knee injury in females as they mature. Interventions to strengthen the knee flexors should target females at the beginning of puberty and emphasized in injury reduction programs aimed at young athletes of both sexes.

\section{KNEE AND ANKLE OVERUSE INJURIES IN YOUTH BASKETBALL PLAYERS}

${ }^{1}$ Carlyn Stilling, 1,20luwatoyosi BA Owoeyea, 'Lauren C Benson, 1,3,4,5 Kati Pasanen, ${ }_{1,2,3,4,6,7}$ Carolyn Emery. 'Sport Injury Prevention Research Centre, Faculty of Kinesiology, University of Calgary, 2500 University Dr., T2N 1N4, Calgary, Canada; ${ }^{2}$ Department of Physical Therapy and Athletic Training, Doisy College of Health Sciences, Saint Louis University, Saint Louis, USA; ${ }^{3}$ Alberta Children's Hospital Research Institute, University of Calgary, Room 293, Heritage Medical Research Building, 3330 Hospital Dr. NW, T2N 4N1, Calgary, Canada; ${ }^{4}$ McCaig Bone and Joint Institute, Cumming School of Medicine, University of Calgary, HRIC 3A08, 3280 Hospital Drive NW, T2N 4Z6, Calgary, Canada; ${ }^{5}$ Tampere Research Center of Sports Medicine, UKK Institute, Kaupinpuistonkatu 1, 33500, Tampere, Finland; ${ }^{6}$ Department of Community Health Sciences, Cumming School of Medicine, University of Calgary, 3D10, 3280 Hospital Drive NW, T2N 4Z6, Calgary, Canada; ${ }^{7}$ Department of Pediatrics, Cumming School of Medicine, University of Calgary, 28 Oki Drive NW, T3B 6A8, Calgary, Canada

\subsection{6/bjsports-2021-IOC.292}

Background Current scientific evidence reveals that overuse injuries may be more prevalent in youth basketball players than previously reported. Many basketball injuries result from gradual onset overuse mechanisms associated with pain, but not resulting in absence from basketball participation.

Objective To determine the season prevalence and burden of all complaint overuse knee injuries (OKIs) and ankle injuries (OAIs) in youth basketball players.

Design Cohort study.

Setting Youth basketball, Calgary, Canada.

Patients (or Participants) A convenience sample of 83 (47F, 36M; Ages 16-18; $\mathrm{N}=7$ ) senior team high school basketball players. 
Interventions (or Assessment of Risk Factors) The Oslo Sports Trauma Research Centre Overuse Injury Questionnaire was used weekly to register OKIs and OAIs over 16 weeks.

Main Outcome Measurements Self-reported OKIs (e.g., patellar tendinopathy, patellofemoral syndrome) and OAIs (e.g., Achilles tendinopathy) and symptom duration.

Results Female teams participated in 52 basketball sessions (range 42-61, SD 8.6, 42\% games) and males in 53 sessions (range 51-54, SD 1.2, 42\% games). In the season, $30.4 \%$ of females and $27.8 \%$ of males reported an OKI and $19.1 \%$ of females and $8.3 \%$ of males an OAI. The median symptom duration (burden) of OKIs was 7 weeks for females and 4 weeks for males. Median time to onset for new OKI cases was 4 weeks for female players and 7 weeks for male players. The median symptom duration of OAIs was 9 weeks for females and 2 weeks for males. Median time to onset for new OAI cases was 3 weeks for females and 7 weeks for males.

Conclusions The seasonal prevalence and symptom duration of OKIs and OAIs is higher in female youth basketball players compared to males. OKIs represent a greater proportion of lower extremity overuse injury in males compared to OAIs. Females reported new OKIs and OAIs earlier in the season compared to males.

\section{SPORT-RELATED CONCUSSION AWARENESS AND KNOWLEDGE AMONG WORLDWIDE SPORTS PHYSICAL THERAPISTS}

1,2,3 Wesam Saleh A Al Attar, ${ }^{1}$ Amir A El Fiky, ${ }^{4}$ Mashaer Alyami, ${ }^{1}$ Ehdaa H Khaledi, ${ }^{1}$ Ahmed Qasem, ${ }^{1}$ Fahad M Alkabkabi, ${ }^{1}$ Nasser G Alshamrani, ${ }^{1}$ Raed S Almalki, ${ }^{1}$ Amirah M Akkam, ${ }^{3}$ Ross H Sanders. 'Department of Physical Therapy, Faculty of Applied Medical Science, Umm Al Qura University, Makkah, Saudi Arabia; ${ }^{2}$ Department of Sport, Exercise and Health, Faculty of Medicine, University of Basel, Basel, Switzerland; ${ }^{3}$ Discipline of Exercise and Sport Science, Faculty of Medicine and Health, The University of Sydney, Sydney, Australia; ${ }^{4}$ King Fahad Specialist Hospital, Dammam, Saudi Arabia

\subsection{6/bjsports-2021-IOC.293}

Background Sport-related concussions (SRC) have gained more attention in the recent years due to its detrimental short-term and long-term effects on the players.

Objective To assess the awareness and knowledge of SRC among sports physical therapists.

Design A cross-sectional study.

Setting An online survey.

Patients (or Participants) A total of 517 sports physical therapists completed the survey.

Interventions (or Assessment of Risk Factors) The survey consisted of multiple-choice questions related to the knowledge of physical therapy in managing SRC patients, case identification, and preventive measures was distributed to sports physical therapists. The questions of the survey were developed using instructions published by the University of Michigan School of Kinesiology Concussion Center (Ann Arbor, MI, USA).

Main Outcome Measurements SRC awareness levels and knowledge among sports physical therapists.

Results The survey scores ranged from $40 \%$ to $100 \%$, with an average score of $62.7 \%$. The highest educational qualification (46\%) recorded among the respondents was a master's degree. There was a difference in the score based on participant qualifications (Welch's F $(2,308.3)=15.3, \mathrm{p}<$ 0.001). Pairwise comparisons revealed that participants with a doctoral/fellowship degree $(62.1 \pm 18.5)$ or a masters' degree $(62.7 \pm 18.5)$ obtained greater scores than participants holding a bachelors' degree $(56.7 \pm 13.8)$ ( $p>0.001)$. Additionally, there was no difference in the score between participants with a doctoral/fellowship degree or a masters' degrees $(p=0.073)$. Furthermore, there was no difference in the survey score based on participants' region (Welch's F (4, 143.3) $=0.08, \mathrm{p}=0.988$ ).

Conclusions The results suggested that a great number of sports physical therapists around the world are aware of current standards and guidelines regarding SRC assessment and management. However, the greatest difference was attributed to higher educational qualification, which denotes its significance recognizing and managing SRC.

\section{ABSTRACT WITHDRAWN}

\section{THE RELATIONSHIP BETWEEN CERVICAL PROPRIOCEPTION AND CONCUSSION IN MALE PROFESSIONAL RUGBY PLAYERS}

${ }^{1,2}$ Theo Farley, ${ }^{1}$ Kath Bester, ${ }^{1}$ Alan Barbero, ${ }^{1}$ Ed Barry, ${ }^{1}$ Jack Thoroughgood,
${ }^{1}$ Richard Sylvester, ${ }^{1}$ Akbar De Medici, 'Mathew Wilson. ${ }^{1}$ The Institute of Sport Exercise and
Health, London, UK; ${ }^{2}$ The English Institute of Sports, London, UK

10.1136/bjsports-2021-IOC.294

Background During a tackle 1997 Newtons of force (equivalent to $204 \mathrm{~kg}$ ) is known to be transmitted to an opponent, however there is currently no known minimal force threshold leading to concussion. Amongst the other factors considered, contact technique has been identified as a potential injury cause, with head position proposed as a key variable.

Objective To investigate whether cervical proprioception is significantly associated with concussion incidence in a group of male professional rugby players.

Design Prospective cohort study.

Setting 165 professional rugby players across 12 Rugby Union teams and three professional leagues were assessed, during the 2018-2019 season.

Interventions Athletes were assessed for cervical proprioception at three time points throughout the season using the Joint Position Error Test.

Main Outcome Measurements Associations with diagnosed concussion injuries are presented as Incidence Rate Ratios (IRR) with $95 \%$ confidence intervals. we present the IRR for a $10 \%$ increase in each variableand compared results against concussion using match minutes to allow for risk exposure.

Results During the study period, 45 concussions were incurred by 44 players giving a rate of 19.7 concussions per 1000 player-match hours. There was a significant association between right rotation repositioning error and concussion with a $5 \%$ increase in concussion risk for each $10 \%$ increase in gross right rotation error $(\mathrm{P}=0.021)$ and a $6 \%$ increase in concussion risk for each $10 \%$ increase in right rotation along the horizontal plane $(\mathrm{P}=0.0001)$.

Conclusions Using the JPET for cervical proprioception it is possible to highlight rugby players who are at greater risk of concussion. The JPET is a fast and cheap test to set up and does not require specialist equipment. 